\title{
A New Watermarking Scheme for an in-Vivo Computer Based on Infection of E. coli
}

\author{
Arash Karimi, Rezvan Dastanian, and Hadi Shahriar Shahhoseini
}

\begin{abstract}
One of the most significant drawbacks that the previously proposed watermarking schemes are suffered from, is size of the watermark picture which should be of limited length. One possible way out of this bottleneck is going into molecular layers. In this regard, We present the first watermarking model in DNA computing. This scheme can be implemented on an in-vivo computer. The proposed scheme uses the bacteriophage lambda genome for coding watermark image (owner-related information) and the Escherichia coli $(E$. coli) bacteria genome for coding the host image. The presented coding scheme is based on the silent mutation of codons which produces harmless bacteria and phages. The significant advantage of this scheme over other methods is that the process of embedding the watermark image in the host image takes place naturally in the process of infecting E. coli cells using bacteriophage lambda in lysogenic cycle. The owner of the watermark image can easily extract his information using the centrifuge technique from the resulting test tube of the proposed scheme. A computer simulation is also conducted in this paper that depicts the processes of coding, embedding and extraction to demonstrate the biological procedures involved in this paper..
\end{abstract}

Index Terms-Bacteriophage lambda, watermark, DNA computing, E. coli bacteria, lysogenic cycle.

\section{INTRODUCTION}

Highlight DNA (deoxyribonucleic acid) is the biochemical basis of heredity in all organisms and recent research has shown that it can be used to solve hard computational problems because of the massive parallelism inherent in DNA molecules and it also has the ability to hide information due to being able to store a large amount of information in a small volume.

Based on the work of Kari et al., the ability of DNA molecules to do computations dates back to millions of years ago when a species of protozoa (ciliates) solved a problem like finding a Hamiltonian path in a graph [1], [2]. Since their work, finding problems which can be solved using in-vivo or in-vitro computers became a popular research area in computing theory community. In [3] a steganography scheme using DNA molecules is proposed. The first leap towards implementation of DNA computers was taken in 2004 by Shapiro et al. [4].

The present proposed idea goes along previous works to find real-world problems posed in the body of a living-being.

Manuscript received May 6, 2012; revised July 27, 2012. This work was supported in part by Iran Telecommunication Research Center (ITRC).

The authors are with the Department of Electrical Engineering Of Iran University of Science and Technology, Narmak, Tehran, 16846-1311, Iran (e-mail: ar_karimi@elec.iust.ac.ir, r_dastanian@elec.iust.ac.ir, hshsh@iust.ac.ir).
It is noteworthy that the proposed ideas so far in this subject are mainly about hiding a text in another text using DNA strands (Steganography). While by the time of writing this paper there is no proposed scheme for image watermarking using biomolecules.

In this paper we use the infection procedure of the E. coli bacteria with phage lambda as our watermark model. The process of embedding the watermark image in the host image consists of two steps. In the first step, the genome of phage lambda is used to encode the owner-related information and the genome of E. coli is utilized to encode the host image. In the second step, two solutions produced by the first step are mixed together for infection of the E. coli bacteria and to produce the resulting image carrying the watermark image. A novel coding algorithm for hiding information into cells of a living-being is also proposed which is based on the multiform property of amino acids.

The rest of this paper is organized as follows. In Section II, the preliminary background is reviewed. Section III gives an overview of the proposed scheme. Section IV describes simulation results. In Section V, Conclusions are drawn.

\section{PReliminary Background}

In this Section for a better understanding of the relationship between the proposed biological cycle with the watermarking scheme, the infection process of E. coli with phage lambda and extraction of phage lambda from the infected bacterium are described.

As can be seen in "Fig. 1", phage lambda first identifies a host E. coli bacterium by binding to a specific structure on the surface of the cell and afterwards it ejects its DNA into the bacterial cell. DNA of lambda then circularizes and next it is cut from cos sites and finds its corresponding sticky ends in bacterial DNA and then it cuts the DNA of E. coli and inserts its DNA into the bacterial DNA. In this regard, a recombinant DNA structure is produced which possesses the combination of lambda DNA and the E. coli DNA. This procedure of infection is called the lysogeny cycle that does not destruct the host cell. There is however, another cycle in which E. coli cells are destructed during the process of infection of the $\mathrm{E}$. coli bacteria. This cycle is called the Lytic cycle which is not of interest of this paper.

To release phage lambda from the infected bacterium, we utilize the Centrifuge method of biotechnology. It is a technique that is based on sedimentation principle in which centripetal acceleration causes more dense substances to be separated along radial direction and go to the bottom of the tube which contains the infected bacteria and lighter particles move to top of the tube. In this regard, since the density of the 
lambda phage is less than that of E. coli, it tends to move to the top of the test tube and we can easily extract DNA of bacteriophage lambda from that of E. coli.

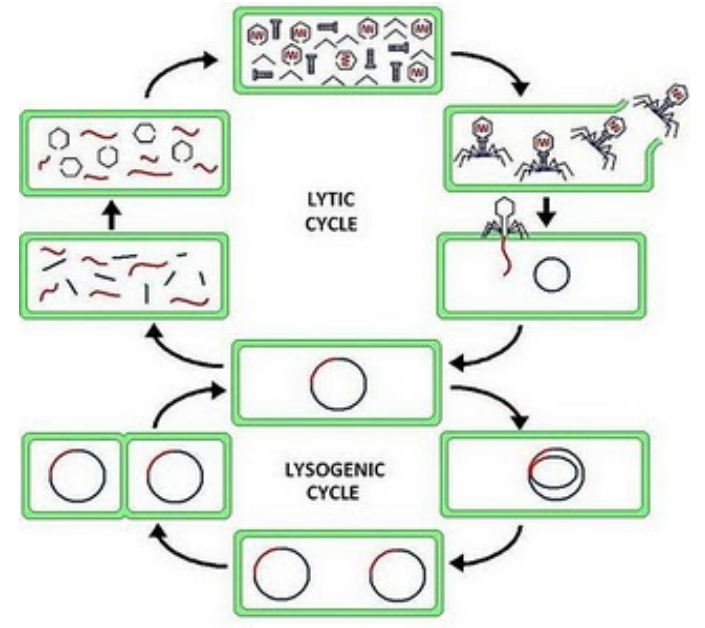

Fig. 1. Lysogenic and lytic cycle

\section{THE Proposed SCHEME}

A remarkable difficulty that is common to all the watermarking algorithms presented up to now is restriction of size of the watermark picture. We have overcome this restriction by using DNA molecules to encode image information into cells of two microbes. Our proposed method has the ability to encode images with a very large size, since the total length of E. coli and phage lambda can be used to encode the host image and the watermark picture respectively (approximately $4000 \times 4000$ pixels for the host image and $127 \times 127$ pixels for the watermark image if we use E. coli and lambda phage for encoding the host image and the watermark image respectively.) Furthermore we can use larger phages to encode larger watermark images. It is noteworthy that for implementation of this scheme in laboratory we should control the infection cycle of E. coli so that it maintains the lysogenic cycle and as shown in [5] these conditions can be achieved with a probability of at least $90 \%$. The proposed scheme like all the other watermarking schemes [6]-[8] includes two steps of embedding the watermark image and extracting the watermark image.

TABLE I: TABLE OF THE GENETIC CODE

\begin{tabular}{|c||c||c|c|c||c|}
\hline \multicolumn{5}{|c|}{ Second position of Codon } \\
\cline { 2 - 6 }
\end{tabular}

\section{A. Embedding Watermark}

In the proposed method, the host image is first converted into a string of sequential bits and then it is mapped to DNA sequence of the bacterium E. coli.

A codon is a 3-tuple of the four possible bases of A, C, T and $\mathrm{G}$ existing in a DNA sequence. There is a total number of 64 possible combinations that can be produced using these letters. There are 20 different amino acids in general. So, some codons may produce one amino acid. As can be seen in table I, codons that produce the same amino acids, are such that their third nucleotides are alike. The mapping of image bits and codons is based on the concept of a Silent mutation which is a kind of mutation that does not alter the amino acid and so does not outbreak in the phenotype.

In the sequel, some Algorithms are brought to explain the detailed procedure of our proposed method.

Algorithm I: Coding of information of the host image in the genome of E. coli

Step1. Selection of the host image.

Step2. Displaying the host image as a sequence of binary bits using the halftone technology [8].

Step3. Selection of a gene from the E. coli genome to possess the size of at least three times as long as the size of bits of the image.

Step4. Coding of the sequence of Step2 in the genome of E. coli such that if its corresponding bit is zero, there will be no change in the structure of the codon of the gene, otherwise, the codon of the corresponding gene incurs a Silent mutation.

In the next step, we should select a specific site in the genes of E. coli and phage lambda that are regarded as the sticky ends of them. It is substantial to note that the sticky ends are unique. Otherwise, by doing it in the laboratory, the circular DNA of E. coli will be patchy and will not yield an appropriate result.

Algorithm II: Coding of information of the watermark
image in the genome of lambda phage
Step1. Selection of the watermark image.
Step2. Displaying the watermark image as a sequence
of binary bits using the halftone technology.
Step3. Selection of a gene from the lambda phage
genome to possess the size of at least three times as long
as the size of bits of the image.
Step4. Coding of the sequence of Step2 in the genome
of phage lambda such that if its corresponding bit is zero,
there will be no change in the structure of the codon of the
gene, otherwise, the codon of the corresponding gene
incurs a Silent mutation.

Algorithms $I$ and $I I$ result in two test tubes that contain the coded DNA strands of the host image and the watermark image respectively. In the next algorithm, an appropriate site must be selected for computer simulation of insertion of the phage DNA into the DNA of E. coli. 
Algorithm III: Selection of the sticky ends of E. coli and phage lambda

Step1. Reception of the genes of E. coli and phage lambda produced in Algorithms I and II.

Step2. Finding a cos site in the phage lambda and the corresponding site in bacteria E. coli.

We have used the cos site achieved in [9] to be used in Step2 of Algorithm III as a secondary attachment site. In the next algorithm, insertion of the phage lambda DNA into the DNA of E. coli is computer simulated.

Algorithm IV: Insertion of the DNA of the phage
lambda in that of E. coli
Step1. Cutting the double-stranded DNA of E.
coli and lambda phage from their sticky ends.
Step2. Insertion of a piece of the lambda phage
into the cutting edge of the E. coli bacteria.

\section{B. The Watermark Image Extraction}

In this stage, to extract the watermark image by the owner he should evict lambda phage from the solution containing the infected E. coli and then he should extract the phage gene and decode it. In Algorithm V, stages of watermark extraction are depicted.

Algorithm V. Evicting phage lambda from the infected
E. coli
Step1. The owner-using the knowledge of sticky ends
of the phage that he himself has inserted-finds two
identical sticky ends and cuts them stepwisely.
Step2. Extraction of a shorter length sequence (phage
lambda).
Step3. Adjoining free nucleotides of the remaining
sequence (E. coli).

In laboratory Algorithm $\mathrm{V}$ is implemented using centrifuge of the resulted solution. In this regard, because lambda phage sequence is shorter than that of E. coli, it moves faster in the test tube and so it can be easily extracted.

Algorithm VI. Decoding
Step1. Comparing sequences of the extracted phage
from step 3 of the algorithm V with the original gene
of the phage to extracting the watermark image.
Step2. Comparing DNA sequence of the resulting
E .coli in step 4 of the algorithm V with the original
sequence of E. coli to extract the host image.

\section{Evaluation}

To show the performance of our proposed scheme we have run a computer simulation in which we want to embed the picture containing name of this conference of size $40 \times 40$ pixels into a $200 \times 200$ pixels Lenna image ("Fig. 2") and next, we will depict the results of our computer simulation in two parts of embedding the watermark and extraction of it.

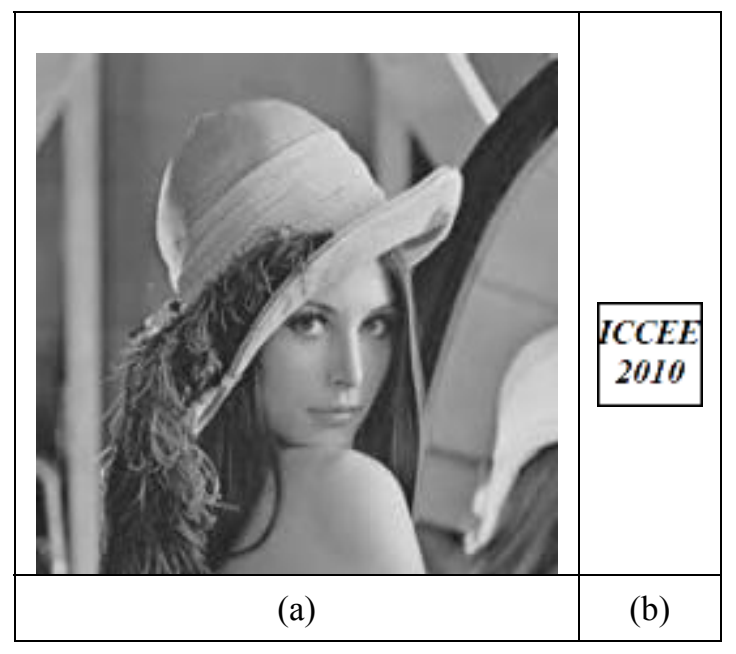

Fig. 2. (a) Host image (b) watermark image

\section{A. Embedding the Watermark}

Our proposed method of embedding watermark is divided into three parts:

1) Coding the host image in a specific gene of E. coli

The host image will be first considered in the halftone mode. i.e. in the dark parts of the image the density of the black pixels will be more and in bright parts of it the density of the black pixels will be less.

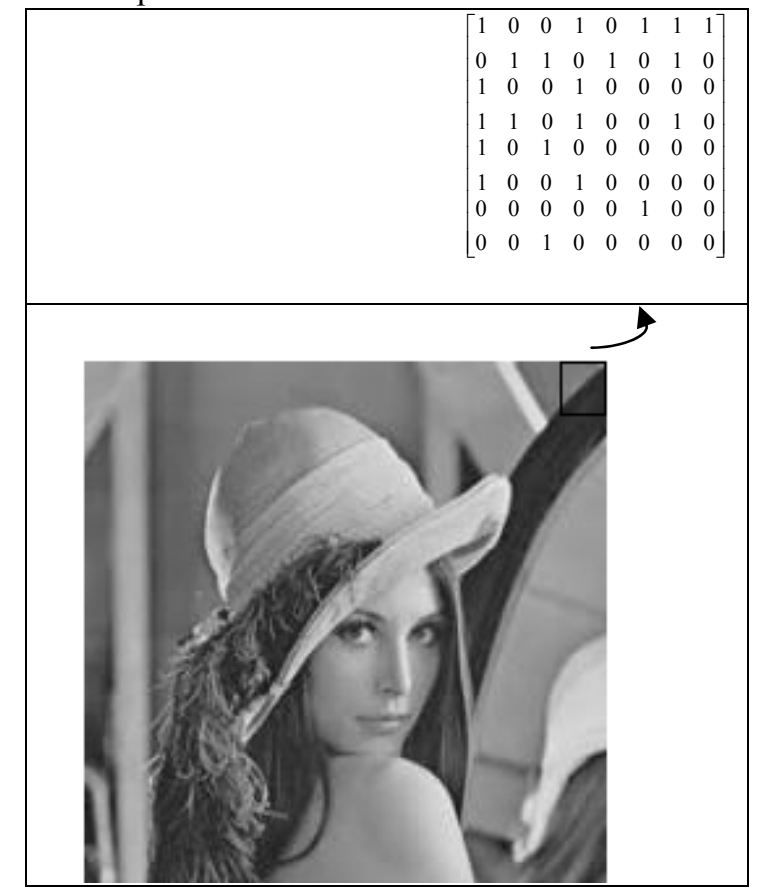

Fig. 3. Host image

Based on the size of the host image, we have chosen gene leuL of E. coli to encode the host image. The part of the abovementioned original gene which corresponds to the selected area demonstrated in "Fig. 3" is presented in matrix of expression (1).

$M_{\text {Se_Ecoli }}=\left[\begin{array}{llllllll}A C C & A A G & C A A & A A A & A C T & A C A & G T T & T C T \\ C C A & C A C & A G T & A A A & C T A & G A C & C T A & A G A \\ C G C & A A A & T T C & T A C & C A A & C T C & A A A & C C C \\ C C G & T G T & C C G & T C A & G C G & G C C & A A G & G C G \\ G C A & T C G & A C T & G C C & G A T & C T A & G T T & T C G \\ C T C & C A T & C T C & T A A & C G G & T A T & G G T & T T G \\ A G G & T T A & G A A & C G A & T T T & C C T & T C G & C C G \\ T T A & A G T & T G G & G G C & A G G & A G A & A A G & C A T\end{array}\right]$


By coding the host image in LeuL, the coded matrix of the selected area of the image is shown in expression (2).

$$
M_{\text {Code_Se_Ecoli }}=\left[\begin{array}{llllllll}
A C G & A A G & C A A & A A G & A C T & A C G & G T G & A G C \\
C C A & C A T & A G C & A A A & C T G & G A C & C T G & A G A \\
A G G & A A A & T T C & T A T & C A A & C T C & A A A & C C C \\
C C T & T G C & C C G & A G C & G C G & G C C & A A A & G C G \\
G C G & T C C & A C G & G C C & G A T & C T A & G T T & T C G \\
C T G & C A T & C T C & T G A & C G G & T A T & G G T & T T G \\
A G G & T T A & G A A & C G A & T T T & C C G & T C G & C C G \\
T T A & A G T & A G C & G G C & A G G & A G A & A A G & C A T
\end{array}\right]
$$

As we can see, if each pixel of the image is one, its corresponding codon will incur a silent mutation (its amino acid does not change) and if the pixel is zero, its corresponding codon will not change.

2) Coding the watermark image in genome of bacteriophage lambda

We use lambda phage as the carrier of our watermark and we encode our watermark image in it. To do so, we should mention that the specific part of the genome of lambda phage should not contain a cos site because the image will be caught from the sticky ends.

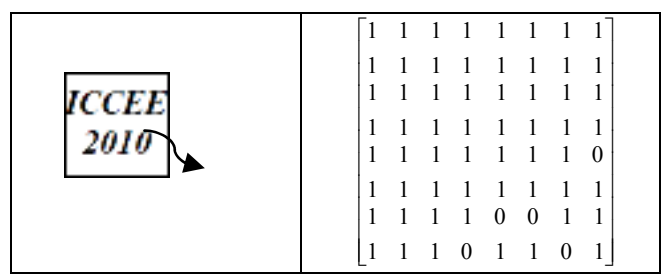

Fig. 4. Watermark image

In the matrix of expression (3) part of phage lambda genome which corresponds to the selected area of the watermark image is shown.

$M_{S_{-} L a m b d a}=\left[\begin{array}{llllllll}T A G & G A A & C T T & C T C & T A T & A T C & A C T & C G G \\ C C C & C G C & T T T & C A A & T G C & A A A & A G C & G A C \\ G A T & C T A & A A G & A T C & T G G & A T C & C T G & T T C \\ A A A & A A G & C G A & C T T & T G G & G G G & G G A & A C G \\ C C G & A T A & G C T & C A G & T G T & T A G & G G A & A T G \\ G T T & G G T & G T C & G T T & A T T & G G T & C A T & C T A \\ C A G & C C G & G A T & A G A & C C A & A A G & G A T & A A G \\ T C T & C G A & T C G & T T T & G G C & A A C & G G A & A A A\end{array}\right]$

By coding the watermark image in lambda phage genome, the coded matrix of the selected area of the image is demonstrated in the matrix of expression (4).

$M_{\text {Code_Se_Lambda }}=\left[\begin{array}{llllllll}T G A & G A G & C T G & C T G & T A C & A T A & A C C & A G G \\ C C G & A G G & T T C & C A G & T G T & A A G & A G G & G A T \\ G A C & C T G & A A A & A T A & A G C & A T A & T T A & T T T \\ A A G & A A A & A G G & C T G & A G C & G G T & G G G & A C T \\ C C T & A T T & G C G & C A A & T G C & T G A & G G G & A T G \\ G T G & G G G & G T G & G T G & A T A & G G G & C A C & C T G \\ C A A & C C T & G A C & A G G & C C A & A A G & G A C & A A A \\ A G C & A G G & A G C & T T T & G G G & A A T & G G A & A A G\end{array}\right]$

3) Infection of escherichia coli with phage lambda

Bacteriophage lambda, in order to infect E. coli, inducts its genome into the TrpC gene of E. coli from its cos site and the recombinant DNA sequence of it is shown in "Fig.5".

ATGCAAACCGTTTTAGCGAAAATCGTCGCAGACAAGGCGATTTGGGTAGAAGCCCGCAAA CAGCAGCAACCGCTGGCCAGTTTTCAGAATGAGGTTCAGCCGAGCACGCGACATTTTTAT GATGCGCTACAGGGTGCGCGCACGGCGTTTATTCTGGAGTGCAAGAAAGCGTCGCCGTCA AAAGGCGTGATCCGTGATGATTTCGATCCAGCACGCATTGCCGCCATTTATAAACATTAC GCTTCGGCAATTTCGGTGCTGACTGATGAGAAATATTTTCAGGGGAGCTTTAATTTCCTC TACCAGATCTATCTGGCGCGCTATTACCAGGCCGATGCCTGCTTATTAATGCTTTCAGTA CTGGATGACGACCAATATCGCCAGCTTGCCGCCGTCGCTCACAGTCTGGAGATGGGGGTG CTGACCGAAGTCAGTAATGAAGAGGAACAGGAGCGCGCCATTGCATTGGGAGCAAAGGTC

Fig. 5. The specific part of the infected E.coli genome
The specific part of the phage genome that carries the cos site is depicted in "Fig.6".

AAATTAGCGCAAGAAGACAAAAATCACCTTGCGCTAATGCTCTGTTACAGGTCACTAATA CCATCTAAGTAGTTGATTCATAGTGACTGCATATGTTGTGTTTT ACAGTATTATGTAGTC TGTTTTTTATGCAAAATCTAATTTAATATATTGATATTTATATCATTTTACGTTTCTCGT TCAGCTTTTTTATACTAAGTTGGCATTATAAAAAAGCATTGCTTATCAATTTGTTGCAAC GAACAGGTCACTATCAGTCAAAATAAAATCATTATTTGATTTCAATTTTGTCCCACTCCC TGCCTCTGTCATCACGATACTGTGATGCCATGGTGTCCGACTTATGCCCGAGAAGATGTT GAGCAAACTTATCGCTTATCTGCTTCTCATAGAGTCTTGCAGACAAACTGCGCAACTCGT GAAAGGTAGGCGGATCCCCTTCGAAGGAAAGACCTGATGCTTTTCGTGCGCGCATAAAAT ACCTTGATACTGTGCCGGATGAAAGCGGTTCGCGACGAGTAGATGCAATTATGGTTTCTC

Fig. 6. The specific part of the lambda bacretiophage genome

The infection procedure of bacteria E. coli with phage lambda and the way its genome enters into the circular DNA of E. coli is demonstrated in" Fig. 7".
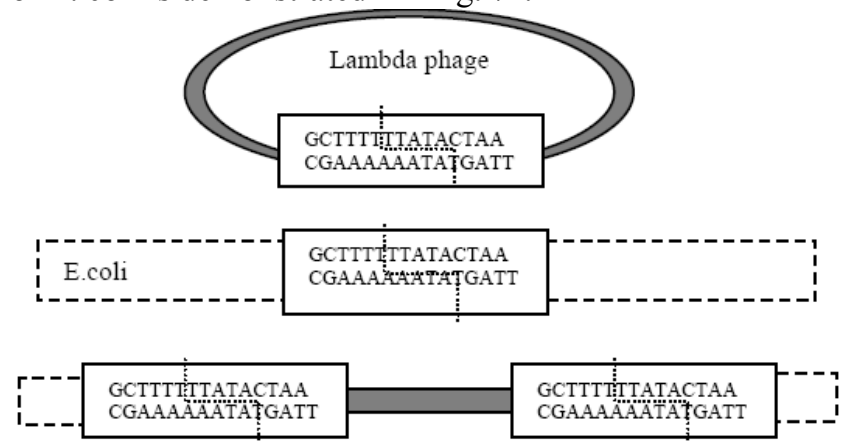

Fig. 7. The infiltration process of lambda phage into E. coli.

\section{B. Watermark Extraction}

In an appropriate watermarking scheme only the owner of the image can extract his watermark and our proposed scheme can meet this need. That is because only the person who embeds the watermark has the knowledge of the genes that the watermark and the host image are hiding into and also, he only knows the sticky ends for selection of the appropriate enzymes to extract the phage. The extraction process of infected E. coli is reverse of process of embedding the watermark image into the host image".

\section{CONCLUSION}

DNA computing is promising for applications that require a large memory and it will supersede the current silicon-based technology in the near future. In this paper a new scheme for watermarking in DNA computing was presented that uses the procedure of infection of E. coli bacteria by lambda bacteriophage in order to embed the watermark image and the utilizes the process of laboratory centrifuge to extract the watermark image out of the watermark embedded image. Furthermore, a new coding method for hiding information into living biological cells is presented which is based on the multiform property of amino acids in the genetic code table and the silent mutation of codons. Our proposed method solves the bottleneck induced by the limitedness of size of the pictures existing in the current watermarking schemes. Our computer simulation results show that this scheme can be easily implemented in the laboratory with accuracy of at least $90 \%$. Of course, there are some drawbacks associated with implementation of this scheme which show up in the laboratory that mainly belong to keeping accurate laboratory circumstances under which the lysogenic cycle for infection procedure of our proposed scheme is maintained and does not turn into the lytic cycle 
(lysogeny induction does not take place) and also we should make sure that the infection procedure succeeds.

\section{ACKNOWLEDGMENT}

The authors are thankful of the financial support provided by Iran Telecommunication Research Center (ITRC). Also, the authors express sincere appreciation to Aazam Mohammad Zadeh for her valuable research assistance for this paper.

\section{REFERENCES}

[1] L. Landweber and L. Kari, "The evolution of cellular computing: nature's solution to a computational problem," LNCS 2950, pp. 207-216, 1998.

[2] L. Kari, "DNA Computing: Arrival of Bilogical Mathematics," The Mathematical Intellignecer, vol. 19, no. 2, pp. 9-22, 1997.

[3] A. Gehani, T. LaBean and J. Reif, "DNA-based Cryptography, Aspects of Molecular Computing," Springer-Verlag Lecture Notes in Computer Science, vol. 2950, 2004.

[4] Y. Benenson, B. Gil, U. B. Dor, R. Adar, and E. Shapiro, "An autonomous molecular computer for logical control of gene expression," Nature 414, pp. 430-434. 2004.

[5] B. A. Fry, "Conditions for the Infection of Escherichia coli with Lambda Phage and for the Establishment of Lysogeny," Journal of gen. Microbiol, vol.21, pp. 676-684, 1959.

[6] C. H. Huang, S. C. Chuang, Y. L. Huang, and J. L. Wu, "Unseen Visible Watermarking: A Novel Methodology for Auxiliary Information Delivery via Visual Contents," IEEE, Trans. On Information Forensics and Security, vol. 4, no. 2, Jun. 2009.

[7] Y. C. Hou, "Visual cryptography for color images," Elsevier, Journal of Pattern Recognition, vol. 36, pp. 1619-1629, 2003.

[8] X. Wang, Z. Xu, and P. Niu, "A feature-based digital watermarking scheme for halftone image," AEU - International Journal of Electronics and Communications, vol. 64, no. 10, pp. 924-933, Oct. 2010.
[9] G. E. Christie and T. Platt, "A secondary attachment site for bacteriophage lambda in trpC of E.coli," Cell, vol. 16, no. 2, pp. 407-413, Feb. 1979.

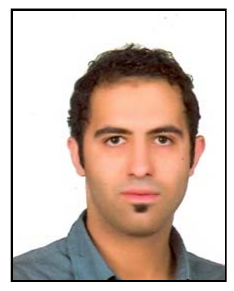

Arash Karimi Received the B.S. and M.S. degrees in the department of electrical engineering from Amirkabir University of Technology (Polytechnic of Tehran) and Iran University of Science and Technology (IUST), Tehran, Iran, in 2008 and 2011, respectively. His research interests include cryptography, unconventional methods in computation with a focus on cryptanalysis, Biochemical computing, and formal languages and automata.

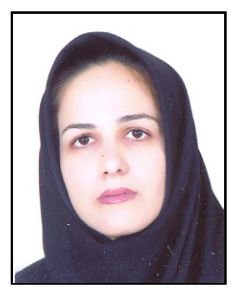

Rezvan Dastanian was born in Iran, Ahvaz, in 1987. She received the B.Sc. and M.Sc. degrees in electrical engineering from Iran University of Science and Technology, Iran, Tehran in 2008 and 2011 respectively and is currently working toward the $\mathrm{Ph} . \mathrm{D}$. degree in electrical engineering at Shiraz University of Technology. Her research interests include cryptography, Biochemical computing, current mode and data converter.

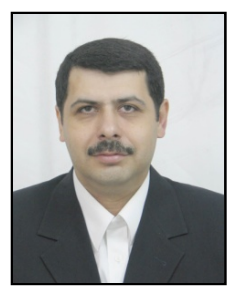

Hadi Shahriar Shahhoseini received B.S. degree in electrical engineering from University of Tehran, in 1990, M.S. degree in electrical engineering from Azad University of Tehran in 1994, and Ph.D. degree in electrical engineering from Iran University of Science and Technology, in 1999. He is an assistant professor of the electrical engineering department in Iran University of Science and Technology. His areas of research include networking, supercomputing and reconfigurable computing. More than 130 papers have been published from his research works in scientific journals and conference proceedings. He is an executive committee member of IEEE TCSC and serves IEEE TCSC as regional coordinator in middle-East Countries. 